\title{
Degree theory and existence of positive solutions to coupled systems of multi-point boundary value problems
}

\author{
Kamal Shah*, Amjad Ali and Rahmat Ali Khan
}

\author{
"Correspondence: \\ kamalshah408@gmail.com \\ Department of Mathematics, \\ University of Malakand, Chakadara \\ Dir(L), Khyber Pakhtunkhwa, \\ Pakistan
}

\begin{abstract}
In this article, we investigate existence and uniqueness of positive solutions to coupled systems of multi-point boundary value problems for fractional order differential equations of the form

$$
\left\{\begin{array}{l}
D^{\alpha} x(t)=\phi(t, x(t), y(t)), \quad t \in I=[0,1], \\
D^{\beta} y(t)=\psi(t, x(t), y(t)), \quad t \in I=[0,1], \\
x(0)=g(x), \quad x(1)=\delta x(\eta), \quad 0<\eta<1, \\
y(0)=h(y), \quad y(1)=\gamma y(\xi), \quad 0<\xi<1,
\end{array}\right.
$$

where $\alpha, \beta \in(1,2], D$ denotes the Caputo fractional derivative, $0<\delta, \gamma<1$ are parameters such that $\delta \eta^{\alpha}<1, \gamma \xi^{\beta}<1, h, g \in C(I, \mathbb{R})$ are boundary functions and $\phi, \psi: / \times \mathbb{R} \times \mathbb{R} \rightarrow \mathbb{R}$ are continuous. We use the technique of topological degree theory to obtain sufficient conditions for existence and uniqueness of positive solutions to the system. Finally, we provide an example to illustrate our main results.
\end{abstract}

MSC: $34 \mathrm{~A} 08 ; 35 \mathrm{R} 11$

Keywords: coupled system; boundary value problems; fractional differential equations; existence and uniqueness of solutions; topological degree theory

\section{空 Springer}

\section{Introduction}

Recently much attention has been paid to investigate sufficient conditions for existence of positive solutions to boundary value problems for fractional order differential equations, we refer to [1-13] and the references therein. This is because of many applications of fractional differential equations in various field of science and technology as in [14-20]. Existence of solutions to boundary value problems for coupled systems of fractional order differential equations has also attracted some attentions, we refer to [13, 20-24]. In these papers, classical fixed point theorems such as Banach contraction principle and Schauder fixed point theorem are used for existence of solutions. The use of these results require stronger conditions on the nonlinear functions involved which restricts the applicability to limited classes of problems and very specialized systems of boundary value problems. In order to enlarge the class of boundary value problems and to impose less restricted conditions, one needs to search for other sophisticated tools of functional analysis. One

(c) 2016 Shah et al. This article is distributed under the terms of the Creative Commons Attribution 4.0 International License (http://creativecommons.org/licenses/by/4.0/), which permits unrestricted use, distribution, and reproduction in any medium, provided you give appropriate credit to the original author(s) and the source, provide a link to the Creative Commons license, and indicate if changes were made. 
such tool is topological degree theory. Few results can be found in the literature which use degree theory arguments for the existence of solutions to boundary value problems (BVPs) [25-32]. However, to the best of our knowledge, the existence and uniqueness of solutions to coupled systems of multi-point boundary value problems for fractional order differential equations with topological degree theory approach have not been studied previously. Wang et al. [28] studied the existence and uniqueness of solutions via topological degree method to a class of nonlocal Cauchy problems of the form

$$
\left\{\begin{array}{l}
D^{q} u(t)=f(t, u(t)), \quad t \in I=[0, T], \\
u(0)+g(u)=u_{0},
\end{array}\right.
$$

where $D^{q}$ is the Caputo fractional derivative of order $q \in(0,1], u_{0} \in \mathbb{R}$, and $f: I \times \mathbb{R} \rightarrow \mathbb{R}$ is continuous. The result was extended to the case of a boundary value problem by Chen $e t$ al. [26] who studied sufficient conditions for existence results for the following two point boundary value problem:

$$
\left\{\begin{array}{l}
D_{0+}^{\alpha} \phi_{p}\left(D_{0+}^{\beta} u(t)\right)=f\left(t, u(t), D_{0+}^{\beta} u(t)\right) \\
D_{0+}^{\beta} u(0)=D_{0+}^{\beta} u(1)=0
\end{array}\right.
$$

where $D_{0+}^{\alpha}$ and $D_{0+}^{\beta}$ are Caputo fractional derivatives, $0<\alpha, \beta \leq 1,1<\alpha+\beta \leq 2$. Wang et al. [27] studied the following two point boundary value problem for fractional differential equations with different boundary conditions:

$$
\left\{\begin{array}{l}
D_{0+}^{\alpha} \phi_{p}\left(D_{0+}^{\beta} u(t)\right)=f\left(t, u(t), D_{0+}^{\beta} u(t)\right), \\
u(0)=0, \quad D_{0+}^{\beta} u(0)=D_{0+}^{\beta} u(1),
\end{array}\right.
$$

where $D_{0+}^{\alpha}$ and $D_{0+}^{\beta}$ are Caputo fractional derivatives, $0<\alpha, \beta \leq 1,1<\alpha+\beta \leq 2$.

Motivated by the work cited above, in this paper, we use a coincidence degree theory approach for condensing maps to obtain sufficient conditions for the existence and uniqueness of solutions to more general coupled systems of nonlinear multi-point boundary value problems. The boundary conditions are also nonlinear. The system is of the form

$$
\left\{\begin{array}{l}
D^{\alpha} x(t)=\phi(t, x(t), y(t)), \quad t \in I=[0,1], \\
D^{\beta} y(t)=\psi(t, x(t), y(t)), \quad t \in I=[0,1], \\
x(0)=g(x), \quad x(1)=\delta x(\eta), \quad 0<\eta<1, \\
y(0)=h(y), \quad y(1)=\gamma y(\xi), \quad 0<\xi<1,
\end{array}\right.
$$

where $\alpha, \beta \in(1,2], D$ is used for standard Caputo fractional derivative and $0<\delta, \gamma<1$ such that $\delta \eta^{\alpha}<1, \gamma \xi^{\beta}<1, h, g \in C(I, \mathbb{R})$ are boundary functions and $\phi, \psi: I \times \mathbb{R} \times \mathbb{R} \rightarrow \mathbb{R}$ are continuous.

\section{Preliminaries}

In this section we give some fundamental definitions and results from fractional calculus and topological degree theory. For further detailed study, we refer to [19, 24, 26, 27, 33, $34]$. 
Definition 2.1 The fractional integral of order $\rho \in \mathbb{R}_{+}$of a function $u \in L^{1}([a, b], \mathbb{R})$ is defined as

$$
I_{0+}^{\rho} u(t)=\frac{1}{\Gamma(\rho)} \int_{a}^{t}(t-s)^{\rho-1} u(s) d s
$$

Definition 2.2 The Caputo fractional order derivative of a function $u$ on the interval $[a, b]$ is defined by

$$
D_{0+}^{\rho} u(t)=\frac{1}{\Gamma(m-\rho)} \int_{a}^{t}(t-s)^{m-\rho-1} u^{(m)}(s) d s
$$

where $m=[\rho]+1$ and $[\rho]$ represents the integer part of $\rho$.

Lemma 2.1 The following result holds for fractional differential equations:

$$
I^{\rho} D^{\rho} u(t)=u(t)+d_{0}+d_{1} t+d_{2} t^{2}+\cdots+d_{m-1} t^{m-1}
$$

for arbitrary $d_{i} \in R, i=0,1,2, \ldots, m-1$.

The spaces $X=C([0,1], \mathbb{R}), Y=C([0,1], \mathbb{R})$ of all continuous functions from $[0,1] \rightarrow$ $\mathbb{R}$ are Banach spaces under the topological norms $\|x\|=\sup \{|x(t)|: t \in[0,1]\}$ and $\|y\|=$ $\sup \{|y(t)|: t \in[0,1]\}$, respectively. The product space $X \times Y$ is a Banach space under the norm $\|(x, y)\|=\|x\|+\|y\|$. It is also a Banach space under the norm $|(x, y)|=\max \{\|x\|,\|y\|\}$.

Let $\mathbb{S}$ be a family of all bounded sets of $\mathfrak{P}(X \times Y)$, where $X \times Y$ is a Banach space. Then we recall the following notions [35].

Definition 2.3 The Kuratowski measure of non-compactness $\mu: \mathbb{S} \rightarrow \mathbb{R}_{+}$is defined as

$$
\mu(S)=\inf \{d>0: S \text { admits a finite cover by sets of diameter } \leq d\},
$$

where $S \in \mathbb{S}$.

Proposition 2.1 The Kuratowski measure $\mu$ satisfies the following properties:

(i) $\mu(S)=0$ iff $S$ is relatively compact.

(ii) $\mu$ is a seminorm, i.e., $\mu(\lambda S)=|\lambda| \mu(S), \lambda \in \mathbb{R}$ and $\mu\left(S_{1}+S_{2}\right) \leq \mu\left(S_{1}\right)+\mu\left(S_{2}\right)$.

(iii) $S_{1} \subset S_{2}$ implies $\mu\left(S_{1}\right) \leq \mu\left(S_{2}\right) ; \mu\left(S_{1} \cup S_{2}\right)=\max \left\{\mu\left(S_{1}\right), \mu\left(S_{2}\right)\right\}$.

(iv) $\mu(\operatorname{conv} S)=\mu(S)$.

(v) $\mu(\bar{S})=\mu(S)$.

Definition 2.4 Let $F: \Omega \rightarrow X$ be continuous bounded map, where $\Omega \subset X$. Then $F$ is $\mu$-Lipschitz if there exists $K \geq 0$ such that

$$
\mu(F(S)) \leq K \mu(S), \quad \forall S \subset \Omega \text { bounded. }
$$

Further, $F$ will be strict $\mu$-contraction if $K<1$. 
Definition 2.5 A function $F$ is $\mu$-condensing if

$$
\mu(F(S))<\mu(S), \quad \forall S \subset \Omega \text { bounded, with } \mu(S)>0 .
$$

In other words, $\mu(F(S)) \geq \mu(S)$ implies $\mu(S)=0$.

Here, we denote the class of all strict $\mu$-contractions $F: \Omega \rightarrow X$ by $\vartheta C_{\mu}(\Omega)$ and denote the class of all $\mu$-condensing maps $F: \Omega \rightarrow X$ by $C_{\mu}(\Omega)$.

Remark $1 \vartheta C_{\mu}(\Omega) \subset C_{\mu}(\Omega)$ and every $F \in C_{\mu}(\Omega)$ is $\mu$-Lipschitz with constant $K=1$.

Moreover, we recall that $F: \Omega \rightarrow X$ is Lipschitz if there exists $K>0$ such that

$$
\|F(x)-F(y)\| \leq K|x-y|, \quad \forall x, y \in \Omega
$$

and that if $K<1$, then $F$ is a strict contraction. For the following results, we refer to [34].

Proposition 2.2 If $F, G: \Omega \rightarrow X$ are $\mu$-Lipschitz with constants $K$ and $K^{\prime}$, respectively, then $F+G: \Omega \rightarrow X$ is $\mu$-Lipschitz with constant $K+K^{\prime}$.

Proposition 2.3 If $F: \Omega \rightarrow X$ is compact, then $F$ is $\mu$-Lipschitz with constant $K=0$.

Proposition 2.4 If $F: \Omega \rightarrow X$ is Lipschitz with constant $K$, then $F$ is $\mu$-Lipschitz with the same constant $K$.

The following theorem due to Isaia [34] plays an important role for our main result.

Theorem 2.1 Let $F: X \rightarrow X$ be $\mu$-condensing and

$$
\Psi=\{x \in X: \exists \lambda \in[0,1] \text { such that } x=\lambda F x\} .
$$

If $\Psi$ is a bounded set in $X$, so there exists $r>0$ such that $\Psi \subset S_{r}(0)$, then the degree

$$
D\left(I-\lambda F, S_{r}(0), 0\right)=1, \quad \forall \lambda \in[0,1] .
$$

Consequently, $F$ has at least one fixed point and the set of the fixed points of $F$ lies in $S_{r}(0)$.

Now, we list the following hypotheses.

$\left(C_{1}\right)$ There exist constants $K_{g}, K_{h} \in[0,1)$ such that

$$
\begin{aligned}
& \left|g\left(x_{2}\right)-g\left(x_{1}\right)\right| \leq K_{g}\left|x_{2}-x_{1}\right|, \\
& \left|h\left(y_{2}\right)-h\left(y_{1}\right)\right| \leq K_{h}\left|y_{2}-y_{1}\right| \quad \text { for } x_{1}, x_{2}, y_{1}, y_{2}, \in \mathbb{R} .
\end{aligned}
$$

$\left(C_{2}\right)$ There exist constants $C_{g}, C_{h}, M_{g}, M_{h}>0$ such that, for $x, y \in \mathbb{R}$,

$$
|g(x)| \leq C_{g}|x|+M_{g}, \quad|h(y)| \leq C_{h}|y|+M_{h}
$$


$\left(C_{3}\right)$ There exist constants $c_{i}, d_{i}(i=1,2)$ and $M_{\phi}, M_{\psi}$ such that, for $x, y \in \mathbb{R}$,

$$
\begin{aligned}
& |\phi(t, x, y)| \leq c_{1}|x|+c_{2}|y|+M_{\phi}, \\
& |\psi(t, x, y)| \leq d_{1}|x|+d_{2}|y|+M_{\psi} .
\end{aligned}
$$

$\left(C_{4}\right)$ There exist constants $L_{\phi}, L_{\psi}$ such that, for $x_{1}, x_{2}, y_{1}, y_{2} \in \mathbb{R}$,

$$
\begin{aligned}
& \left|\phi\left(t, x_{2}, y_{2}\right)-\phi\left(t, x_{1}, y_{1}\right)\right| \leq L_{\phi}\left(\left|x_{2}-x_{1}\right|+\left|y_{2}-y_{1}\right|\right), \\
& \left|\psi\left(t, x_{2}, y_{2}\right)-\psi\left(t, x_{1}, y_{1}\right)\right| \leq L_{\psi}\left(\left|x_{2}-x_{1}\right|+\left|y_{2}-y_{1}\right|\right) .
\end{aligned}
$$

\section{Main results}

In this section, we discuss the existence and uniqueness of solutions to the BVP (1).

Lemma 3.1 If $h: I \rightarrow \mathbb{R}$ is $\alpha$ times integrable function, then solutions of the BVP

$$
\begin{array}{ll}
D^{\alpha} x(t)=h(t), & t \in I=[0,1], \\
x(0)=g(x), & x(1)=\delta x(\eta), \quad 0<\eta<1,
\end{array}
$$

are a solution of the following Fredholm integral equation:

$$
x(t)=\left(1-\frac{t(1-\delta)}{1-\delta \eta}\right) g(x)+\int_{0}^{1} G_{\alpha}(t, s) h(s) d s, \quad t \in[0,1]
$$

where $G_{\alpha}(t, s)$ is defined by

$$
G_{\alpha}(t, s)=\frac{1}{\Gamma(\alpha)} \begin{cases}(t-s)^{\alpha-1}+\frac{t \delta(\eta-s)^{\alpha-1}}{1-\delta \eta}-\frac{t(1-s)^{\alpha-1}}{1-\delta \eta}, & 0 \leq s \leq t \leq \eta \leq 1, \\ (t-s)^{\alpha-1}-\frac{t(1-s)^{\alpha-1}}{1-\delta \eta}, & 0 \leq \eta \leq s \leq t \leq 1 \\ \frac{t \delta(\eta-s)^{\alpha-1}}{1-\delta \eta}-\frac{t(1-s)^{\alpha-1}}{1-\delta \eta}, & 0 \leq t \leq s \leq \eta \leq 1 \\ -\frac{t(1-s)^{\alpha-1}}{1-\delta \eta}, & 0 \leq \eta \leq t \leq s \leq 1\end{cases}
$$

Proof Applying $I^{\alpha}$ on $D^{\alpha} x(t)=h(t)$ and using Lemma 2.1, we have

$$
x(t)=I^{\alpha} h(t)+c_{0}+c_{1} t
$$

for some $c_{0}, c_{1} \in R$. The conditions $x(0)=g(x)$ and $x(1)=\delta x(\eta)$ imply $c_{0}=g(x)$ and $c_{1}=$ $\frac{\delta}{1-\delta \eta} I^{\alpha} h(\eta)-\frac{1-\delta}{1-\delta \eta} g(x)-\frac{1}{1-\delta \eta} I^{\alpha} h(1)$. Hence, we obtain

$$
x(t)=I^{\alpha} h(t)+g(x)+t\left[\frac{\delta}{1-\delta \eta} I^{\alpha} h(\eta)-\frac{1-\delta}{1-\delta \eta} g(x)-\frac{1}{1-\delta \eta} I^{\alpha} h(1)\right]
$$

which after rearranging can be written as

$$
x(t)=\left(1-\frac{t(1-\delta)}{1-\delta \eta}\right) g(x)+\int_{0}^{1} G_{\alpha}(t, s) h(s) d s .
$$


In view of Lemma 3.1, solutions of the coupled systems of BVPs (1) are solutions of the following coupled systems of Fredholm integral equations:

$$
\left\{\begin{array}{lc}
x(t)=\left(1-\frac{t(1-\delta)}{1-\delta \eta}\right) g(x)+\int_{0}^{1} G_{\alpha}(t, s) \phi(t, x(s), y(s)) d s, & t \in[0,1], \\
y(t)=\left(1-\frac{t(1-\gamma)}{1-\gamma \xi}\right) h(y)+\int_{0}^{1} G_{\beta}(t, s) \psi(t, x(s), y(s)) d s, & t \in[0,1]
\end{array}\right.
$$

where $G_{\beta}(t, s)$ is defined by

$$
G_{\beta}(t, s)=\frac{1}{\Gamma(\beta)} \begin{cases}(t-s)^{\beta-1}+\frac{t \gamma(\xi-s)^{\beta-1}}{1-\gamma \xi}-\frac{t(1-s)^{\beta-1}}{1-\gamma \xi}, & 0 \leq s \leq t \leq \xi \leq 1, \\ (t-s)^{\beta-1}-\frac{t(1-s)^{\beta-1}}{1-\gamma \xi}, & 0 \leq \xi \leq s \leq t \leq 1, \\ \frac{t \gamma(\xi-s)^{\beta-1}}{1-\gamma \xi}-\frac{t(1-s)^{\beta-1}}{1-\gamma \xi}, & 0 \leq t \leq s \leq \xi \leq 1, \\ -\frac{t(1-s)^{\beta-1}}{1-\gamma \xi}, & 0 \leq \xi \leq t \leq s \leq 1 .\end{cases}
$$

Clearly

$$
\begin{aligned}
& \max _{t \in[0,1]}\left|G_{\alpha}(t, s)\right|=\frac{(1-s)^{\alpha-1}}{(1-\delta \eta) \Gamma(\alpha)}, \\
& \max _{t \in[0,1]}\left|G_{\beta}(t, s)\right|=\frac{(1-s)^{\beta-1}}{(1-\gamma \xi) \Gamma(\beta)}, \quad s \in[0,1] .
\end{aligned}
$$

Define the operators $F_{1}: X \rightarrow X, F_{2}: Y \rightarrow Y$ by

$$
\begin{aligned}
& F_{1}(x)(t)=\left(1-\frac{t(1-\delta)}{1-\delta \eta}\right) g(x), \\
& F_{2}(y)(t)=\left(1-\frac{t(1-\gamma)}{1-\gamma \xi}\right) h(y)
\end{aligned}
$$

and the operators $G_{1}, G_{2}: X \times Y \rightarrow X \times Y$ by

$$
\begin{aligned}
& G_{1}(x, y)(t)=\int_{0}^{1} G_{\alpha}(t, s) \phi(t, x(s), y(s)) d s \\
& G_{2}(x, y)(t)=\int_{0}^{1} G_{\beta}(t, s) \psi(t, x(s), y(s)) d s .
\end{aligned}
$$

Further, we define $F=\left(F_{1}, F_{2}\right), G=\left(G_{1}, G_{2}\right)$ and $T=F+G$. Then the system of integral equations (7) can be written as an operator equation of the form

$$
(x, y)=T(x, y)=F(x, y)+G(x, y)
$$

and solutions of the system (7) are fixed points of $T$.

Lemma 3.2 Under the assumptions $\left(\mathrm{C}_{1}\right)$ and $\left(\mathrm{C}_{2}\right)$, the operator $F$ satisfies the Lipschitz condition and the following growth condition:

$$
\|F(x, y)\| \leq C\|(x, y)\|+M \quad \text { for every }(x, y) \in X \times Y .
$$


Proof Using the assumption $\left(C_{1}\right)$, we obtain

$$
\begin{aligned}
& |F(x, y)(t)-F(u, v)(t)| \\
& \quad=\left|\left(1-\frac{t(1-\delta)}{1-\delta \eta}\right)(g(x)-g(u))+\left(1-\frac{t(1-\gamma)}{1-\gamma \xi}\right)(h(y)-h(v))\right| \\
& \quad \leq K_{g}\|x-u\|+K_{h}\|y-v\| \leq K\|(x, y)-(u, v)\|, \quad K=\max \left\{K_{g}, K_{h}\right\} .
\end{aligned}
$$

By Proposition 2.4, $F$ is also $\mu$-Lipschitz with constant $K$. Now for the growth condition using $\left(\mathrm{C}_{2}\right)$, we get

$$
\|F(x, y)\|=\left\|\left(F_{1}(x), F_{2}(y)\right)\right\|=\left\|F_{1}(x)\right\|+\left\|F_{2}(y)\right\| \leq C\|(x, y)\|+M,
$$

where $C=\max \left\{C_{g}, C_{h}\right\}, M=\max \left\{M_{g}, M_{h}\right\}$.

Lemma 3.3 The operator $G$ is continuous and under the assumption $\left(\mathrm{C}_{3}\right)$ satisfies the growth condition

$$
\|G(x, y)\| \leq \Delta\|(x, y)\|+\Lambda \quad \text { for every }(x, y) \in X \times Y
$$

where $\Delta=\theta(c+d), \theta=\max \left\{\frac{1}{(1-\delta \eta) \Gamma(\alpha)}, \frac{1}{(1-\gamma \xi) \Gamma(\beta)}\right\}, c=\max \left\{c_{1}, c_{2}\right\}, d=\max \left\{d_{1}, d_{2}\right\}, \Lambda=$ $\theta\left(M_{\phi}+M_{\psi}\right)$.

Proof Let $\left\{\left(x_{n}, y_{n}\right)\right\}$ be a sequence of a bounded set $U_{r}=\{\|(x, y)\| \leq r:(x, y) \in X \times Y\}$ such that $\left(x_{n}, y_{n}\right) \rightarrow(x, y)$ in $U_{r}$. We need to prove that $\left\|G\left(x_{n}, y_{n}\right)-G(x, y)\right\| \rightarrow 0$. Consider

$$
\begin{aligned}
&\left|G_{1}\left(x_{n}, y_{n}\right)(t)-G_{1}(x, y)(t)\right| \\
& \leq \frac{1}{\Gamma(\alpha)}\left[\int_{0}^{t}(t-s)^{\alpha-1}\left|\phi\left(s, x_{n}(s), y_{n}(s)\right)-\phi(s, x(s), y(s))\right| d s\right. \\
& \quad+\frac{\delta}{1-\delta \eta} \int_{0}^{\eta}(\eta-s)^{\alpha-1}\left|\phi\left(s, x_{n}(s), y_{n}(s)\right)-\phi(s, x(s), y(s))\right| d s \\
&\left.\quad+\frac{1}{1-\delta \eta} \int_{0}^{1}(1-s)^{\alpha-1}\left|\phi\left(s, x_{n}(s), y_{n}(s)\right)-\phi(s, x(s), y(s))\right| d s\right] .
\end{aligned}
$$

From the continuity of $\phi$ and $\psi$, it follows that $\phi\left(s, x_{n}(s), y_{n}(s)\right) \rightarrow \phi(s, x(s), y(s))$ as $n \rightarrow \infty$. For each $t \in I$, using $\left(C_{3}\right)$, we obtain $(t-s)^{\alpha-1}\left|\phi\left(s, x_{n}, y_{n}\right)-\phi(s, x, y)\right| \leq(t-s)^{\alpha-1} 2\left(\left(c_{1}+c_{2}\right) r+\right.$ $\left.M_{\phi}\right)$, which implies the integrability for $s, t \in I$ and by using the Lebesgue dominated convergence theorem, we obtain $\int_{0}^{t}(t-s)^{\alpha-1}\left|\phi\left(s, x_{n}, y_{n}\right)-\phi(s, x, y)\right| d s \rightarrow 0$ as $n \rightarrow \infty$. Similarly, the other terms approach 0 as $n \rightarrow \infty$. It follows that

$$
\left\|G_{1}\left(x_{n}, y_{n}\right)(t)-G_{1}(x, y)(t)\right\| \rightarrow 0 \quad \text { as } n \rightarrow \infty
$$

and similarly we can show that

$$
\left\|G_{2}\left(x_{n}, y_{n}\right)(t)-G_{2}(x, y)(t)\right\| \rightarrow 0 \quad \text { as } n \rightarrow \infty
$$


Now for growth condition on $G$, using $\left(C_{3}\right)$ and (9), we obtain

$$
\left|G_{1}(x, y)(t)\right|=\left|\int_{0}^{1} G_{\alpha}(t, s) \phi(s, x(s), y(s)) d s\right| \leq \frac{1}{(1-\delta \eta) \Gamma(\alpha)}\left(c_{1}\|x\|+c_{2}\|y\|+M_{\phi}\right)
$$

and

$$
\left|G_{2}(x, y)(t)\right|=\left|\int_{0}^{1} G_{\beta}(s, t) \psi(s, x(s), y(s)) d s\right| \leq \frac{1}{(1-\gamma \xi) \Gamma(\beta)}\left(d_{1}\|x\|+d_{2}\|y\|+M_{\psi}\right) .
$$

Hence, it follows that

$$
\begin{aligned}
\|G(x, y)\| & =\left\|G_{1}(x, y)\right\|+\left\|G_{2}(x, y)\right\| \\
& \leq \theta\left(c_{1}\|x\|+c_{2}\|y\|+M_{\phi}\right)+\theta\left(d_{1}\|x\|+d_{2}\|y\|+M_{\psi}\right) \\
& \leq \theta(c+d)(\|x\|+\|y\|)+\theta\left(M_{\phi}+M_{\psi}\right)=\Delta\|(x, y)\|+\Lambda .
\end{aligned}
$$

By this, we complete the proof.

Lemma 3.4 The operator $G: X \times Y \rightarrow X \times Y$ is compact. Consequently, $G$ is $\mu$-Lipschitz with zero constant.

Proof Take a bounded set $\mathcal{B} \subset U_{r} \subseteq X \times Y$ and a sequence $\left\{\left(x_{n}, y_{n}\right)\right\}$ in $\mathcal{B}$, then using (13), we have

$$
\left\|G\left(x_{n}, y_{n}\right)\right\| \leq \Delta r+\Lambda \quad \text { for every }(x, y) \in X \times Y,
$$

which implies that $G(\mathcal{B})$ is bounded. Now, for equi-continuity and for given $\epsilon>0$, take

$$
\delta=\min \left\{\delta_{1}=\frac{1}{2}\left(\frac{\epsilon \Gamma(\alpha+1)}{6\left(\left[c_{1}+c_{2}\right] r+M_{\phi}\right)}\right)^{\frac{1}{\alpha}}, \delta_{2}=\frac{1}{2}\left(\frac{\epsilon \Gamma(\beta+1)}{6\left(\left[d_{1}+d_{2}\right] r+M_{\psi}\right)}\right)^{\frac{1}{\beta}}\right\} .
$$

For each $\left(x_{n}, y_{n}\right) \in \mathcal{B}$, we claim that if $t, \tau \in[0,1]$ and $0<\tau-t<\delta_{1}$, then

$$
\left|G_{1}\left(x_{n}, y_{n}\right)(t)-G_{1}\left(x_{n}, y_{n}\right)(\tau)\right|<\frac{\epsilon}{2} .
$$

Now consider

$$
\begin{aligned}
& \left|G_{1}\left(x_{n}, y_{n}\right)(t)-G_{1}\left(x_{n}, y_{n}\right)(\tau)\right| \\
& =\mid \frac{1}{\Gamma(\alpha)} \int_{0}^{t}\left[(t-s)^{\alpha-1}-(\tau-s)^{\alpha-1}\right] \phi\left(s, x_{n}(s), y_{n}(s)\right) d s \\
& \quad+\frac{1}{\Gamma(\alpha)} \int_{t}^{\tau}(\tau-s)^{\alpha-1} \phi\left(s, x_{n}(s), y_{n}(s)\right) d s \\
& \quad+\frac{\delta(t-\tau)}{(1-\delta \eta) \Gamma(\alpha)} \int_{0}^{\eta}(\eta-s)^{\alpha-1} \phi\left(s, x_{n}(s), y_{n}(s)\right) d s \\
& \quad+\frac{(\tau-t)}{(1-\delta \eta) \Gamma(\alpha)} \int_{0}^{1}(1-s)^{\alpha-1} \phi\left(s, x_{n}(s), y_{n}(s)\right) d s \mid
\end{aligned}
$$




$$
\begin{aligned}
& \leq \frac{\left(c_{1}\left|x_{n}\right|+c_{2}\left|y_{n}\right|+M_{\phi}\right)}{\Gamma(\alpha+1)}\left[\left(t^{\alpha}-\tau^{\alpha}\right)+2(\tau-t)^{\alpha}\right] \\
& \leq \frac{\left(c_{1}+c_{2}\right) r+M_{\phi}}{\Gamma(\alpha+1)}\left[\left(\tau^{\alpha}-t^{\alpha}\right)+2(\tau-t)^{\alpha}\right] .
\end{aligned}
$$

We continue the proof with several cases.

Case 1. $\delta_{1} \leq t<\tau<1$ :

$$
\begin{aligned}
\left|G_{1}\left(x_{n}, y_{n}\right)(t)-G_{1}\left(x_{n}, y_{n}\right)(\tau)\right| & <\frac{\left(c_{1}+c_{2}\right) r+M_{\phi}}{\Gamma(\alpha+1)}(2+\alpha) \delta_{1}^{\alpha-1}(\tau-t) \\
& <\frac{\left(c_{1}+c_{2}\right) r+M_{\phi}}{\Gamma(\alpha+1)}(2+\alpha) \delta_{1}^{\alpha}<\frac{\epsilon}{2} .
\end{aligned}
$$

Case 2. $0 \leq t<\delta_{1}, \tau<2 \delta_{1}$ :

$$
\begin{aligned}
\left|G_{1}\left(x_{n}, y_{n}\right)(t)-G_{1}\left(x_{n}, y_{n}\right)(\tau)\right| & <\frac{\left(c_{1}+c_{2}\right) r+M_{\phi}}{\Gamma(\alpha+1)}\left[3 \tau^{\alpha}\right] \\
& <\frac{\left(c_{1}+c_{2}\right) r+M_{\phi}}{\Gamma(\alpha+1)} 3\left(2 \delta_{1}\right)^{\alpha}=\frac{\epsilon}{2} .
\end{aligned}
$$

Similarly for the second part, for each $\left(x_{n}, y_{n}\right) \in \mathcal{B}$, we claim that if $t, \tau \in[0,1]$ and $0<$ $\tau-t<\delta_{2}$, then $\left|G_{2}\left(x_{n}, y_{n}\right)(t)-G_{2}\left(x_{n}, y_{n}\right)(\tau)\right|<\frac{\epsilon}{2}$. Then:

Case 1. $\delta_{2} \leq t<\tau<1$ :

$$
\begin{aligned}
\left|G_{2}\left(x_{n}, y_{n}\right)(t)-G_{2}\left(x_{n}, y_{n}\right)(\tau)\right| & <\frac{\left(d_{1}+d_{2}\right) r+M_{\psi}}{\Gamma(\beta+1)}(2+\beta) \delta_{2}^{\beta-1}(\tau-t) \\
& <\frac{\left(d_{1}+d_{2}\right) r+M_{\psi}}{\Gamma(\beta+1)}(2+\beta) \delta_{2}^{\beta}<\frac{\epsilon}{2} .
\end{aligned}
$$

Case $2.0 \leq t<\delta_{2}, \tau<2 \delta_{2}$ :

$$
\begin{aligned}
\left|G_{2}\left(x_{n}, y_{n}\right)(t)-G_{2}\left(x_{n}, y_{n}\right)(\tau)\right| & <\frac{\left(d_{1}+d_{2}\right) r+M_{\psi}}{\Gamma(\beta+1)}\left[3 \tau^{\beta}\right] \\
& <\frac{\left(d_{1}+d_{2}\right) r+M_{\psi}}{\Gamma(\beta+1)} 3\left(2 \delta_{2}\right)^{\beta}=\frac{\epsilon}{2} .
\end{aligned}
$$

Hence, we have

$$
\left\|G\left(x_{n}, y_{n}\right)-G\left(x_{n}, y_{n}\right)\right\|<\epsilon .
$$

Thus $G(\mathcal{B})$ is equi-continuous. In view of the Arzelà-Ascoli theorem $G(\mathcal{B})$ is compact. Furthermore, by Proposition 2.3, $G$ is $\mu$-Lipschitz with constant zero.

Theorem 3.1 Under the assumptions $\left(C_{1}\right)-\left(C_{3}\right)$, the system (1) has at least one solution $(x, y) \in X \times Y$ provided $C+\Delta<1$. Moreover, the set of solutions of (1) is bounded in $X \times Y$.

Proof By Lemma 3.2, $F$ is $\mu$-Lipschitz with constant $K \in[0,1)$ and by Lemma 3.4 $G$ is $\mu$-Lipschitz with constant 0 . It follows by Proposition 2.2 that $T$ is a strict $\mu$-contraction with constant $K$. Define

$$
\mathbb{B}=\{(x, y) \in X \times Y: \text { there exist } \lambda \in[0,1] \text { such that }(x, y)=\lambda T(x, y)\} .
$$


We have to prove that $\mathbb{B}$ is bounded in $X \times Y$. For this, choose $(x, y) \in \mathbb{B}$, then in view of the growth conditions as in Lemmas 3.2 and 3.3, we have

$$
\begin{aligned}
\|(x, y)\| & =\|\lambda T(x, y)\|=\lambda\|T(x, y)\| \leq \lambda(\|F(x, y)\|+\|G(x, y)\|) \\
& \leq \lambda[C\|(x, y)\|+M+\Delta\|(x, y)\|+\Lambda] \\
& =\lambda(C+\Delta)\|(x, y)\|+\lambda(M+\Lambda)
\end{aligned}
$$

which implies that $\mathbb{B}$ is bounded in $X \times Y$. Therefore, by Theorem 2.1, $T$ has at least one fixed point and the set of fixed points is bounded in $X \times Y$.

Theorem 3.2 In addition to the assumption $\left(\mathrm{C}_{1}\right)-\left(\mathrm{C}_{4}\right)$, assume that $K+\theta\left(L_{\phi}+L_{\psi}\right)<1$, then the system (1) has a unique solution.

Proof We use the Banach contraction theorem, for $(x, y),(u, v) \in \mathbb{R} \times \mathbb{R}$, we have from (12)

$$
|F(x, y)-F(u, v)| \leq K\|(x, y)-(u, v)\| .
$$

Using $\left(\mathrm{C}_{4}\right)$ and (9), we obtain

$$
\begin{aligned}
\left|G_{1}(x, y)-G_{1}(u, v)\right| & \leq \int_{0}^{1}\left|G_{\alpha}(t, s)\right||\phi(s, x(s), y(s))-\phi(s, u(s), v(s))| d s \\
& \leq \theta L_{\phi}(|x-u|+|y-v|),
\end{aligned}
$$

which implies that

$$
\begin{aligned}
\left\|G_{1}(x, y)-G_{1}(u, v)\right\| & \leq \theta L_{\phi}(\|x-u\|+\|y-v\|) \\
& =\theta L_{\phi}\|(x-u, y-v)\| \\
& =\theta L_{\phi}\|(x, y)-(u, v)\| .
\end{aligned}
$$

Similarly, we have

$$
\left\|G_{2}(x, y)-G_{2}(u, v)\right\| \leq \theta L_{\psi}\|(x, y)-(u, v)\| .
$$

From (16) and (17), it follows that

$$
\begin{aligned}
\|G(x, y)-G(u, v)\| & =\left\|G_{1}(x, y)-G_{1}(u, v)\right\|+\left\|G_{2}(x, y)-G_{2}(u, v)\right\| \\
& \leq \theta\left(L_{\psi}+L_{\psi}\right)\|(x, y)-(u, v)\| .
\end{aligned}
$$

Hence, in view of (15) and (18), we obtain

$$
\begin{aligned}
|T(x, y)-T(u, v)| & \leq\|F(x, y)-F(u, v)\|+\|G(x, y)-G(u, v)\| \\
& \leq\left(K+\theta\left(L_{\psi}+L_{\psi}\right)\right)\|(x, y)-(u, v)\|,
\end{aligned}
$$

which implies that $T$ is a contraction. By the Banach contraction principle, the system (1) has a unique solution. 


\section{Example}

Example 4.1 Consider the following multi-point BVP:

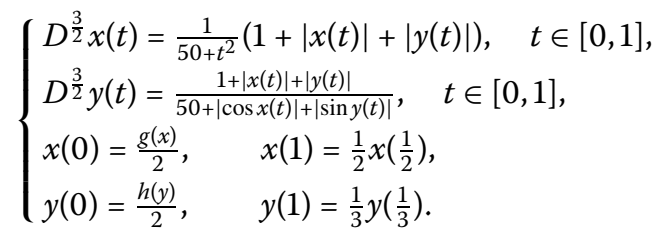

The solution of the BVP (19) is given by

$$
\begin{aligned}
& x(t)=\frac{g(x)}{2}\left(1-\frac{2 t}{3}\right)+\int_{0}^{1} G_{\alpha}(t, s) \phi(s, x(s), y(s)) d s, \\
& y(t)=\frac{h(y)}{2}\left(1-\frac{3 t}{4}\right)+\int_{0}^{1} G_{\beta}(t, s) \psi(s, x(s), y(s)) d s,
\end{aligned}
$$

where $G_{\alpha}, G_{\beta}$ are the Green's functions and can be obtained easily as obtained generally in (4) and (8), respectively. From the system (19) we take $\alpha=\beta=\frac{3}{2}, \delta=\eta=\frac{1}{2}, \gamma=\xi=\frac{1}{3}$, and $r=2 \in(1,3)$, and let us take $\lambda=\frac{1}{2} \in[0,1]$. Then by the use of Theorem 3.2, we have $L_{\phi}=$ $L_{\psi}=\frac{1}{50}, M_{\phi}=M_{\psi}=\frac{1}{50}=c_{i}=d_{i}$, for $i=1,2$, and taking $K_{g}=\frac{1}{2}, K_{h}=\frac{1}{2}$, then assumptions $\left(\mathrm{C}_{1}\right)-\left(\mathrm{C}_{4}\right)$ are satisfied. We have

$$
\begin{array}{ll}
F_{1} x(t)=\frac{g(x)}{2}\left(1-\frac{2 t}{3}\right), & G_{1} x(t)=\int_{0}^{1} G_{\alpha}(t, s) \phi(s, x(s), y(s)) d s, \\
F_{2} y(t)=\frac{h(y)}{2}\left(1-\frac{3 t}{4}\right), & G_{2} y(t)=\int_{0}^{1} G_{\beta}(t, s) \psi(s, x(s), y(s)) d s .
\end{array}
$$

Since $F_{1}, F_{2}, G_{1}, G_{2}$ are continuous and bounded, also $F=\left(F_{1}, F_{2}\right), G=\left(G_{1}, G_{2}\right)$, which further implies that $T=F+G$ is continuous and bounded. Further

$$
\|F(x, y)-F(u, v)\| \leq \frac{1}{2}\|(x, y)-(u, v)\|,
$$

that is, if $F$ is $\mu$-Lipschitz with Lipschitz constant $\frac{1}{2}$ and $G$ is $\mu$-Lipschitz with zero constant, this implies that $T$ is a strict- $\mu$-contraction with constant $\frac{1}{2}$. Further it is easy to calculate $\theta=1.5045$. As

$$
\mathbb{B}=\left\{(x, y) \in C(I \times \mathbb{R} \times \mathbb{R}, \mathbb{R}), \exists \lambda \in[0,1]:(x, y)=\frac{1}{2} T(x, y)\right\} .
$$

Then the solution

$$
\|(x, y)\| \leq \frac{1}{2}\|T(x, y)\| \leq 1,
$$

implies that $\mathbb{B}$ is bounded and by Theorem 3.1 the BVP (19) has at least a solution $(x, y)$ in $C(I \times \mathbb{R} \times \mathbb{R}, \mathbb{R})$. Furthermore $K+\theta\left(L_{\phi}+L_{\psi}\right)=0.56018<1$. Hence by Theorem 3.2 the boundary value problem (19) has a unique solution. 
Authors' contributions

The authors have equally made contributions. All authors read and approved the final version of this manuscript.

\section{Acknowledgements}

We are thankful to the reviewver for his/her valuable suggestions, which improved the manuscript.

\section{Received: 6 November 2015 Accepted: 4 February 2016 Published online: 11 February 2016}

\section{References}

1. Agarwal, RP, Benchohra, M, Hamani, S: A survey on existence results for boundary value problems of nonlinear fractional differential equations and inclusions. Acta Appl. Math. 109, 973-1033 (2010)

2. Agarwal, RP, Belmekki, M, Benchohra, M: A survey on semilinear differential equations and inclusions involving Riemann-Liouville fractional derivative. Adv. Differ. Equ. 2009, Article ID 981728 (2009)

3. Balachandran, K, Kiruthika, S, Trujillo, JJ: Existence results for fractional impulsive integrodifferential equations in Banach spaces. Commun. Nonlinear Sci. Numer. Simul. 16, 1970-1977 (2011)

4. Benchohra, M, Graef, JR, Hamani, S: Existence results for boundary value problems with nonlinear fractional differential equations. Appl. Anal. 87, 851-863 (2008)

5. El-Sayed, AMA, Bin-Taher, EO: Positive solutions for a nonlocal multi-point boundary-value problem of fractional and second order. Electron. J. Differ. Equ. 2013, 64 (2013)

6. El-Shahed, M, Nieto, JJ: Nontrivial solutions for a nonlinear multi-point boundary value problem of fractional order. Comput. Math. Appl. 59(11), 3438-3443 (2010)

7. Han, $\mathrm{X}$, Wang, $\mathrm{T}$ : The existence of solutions for a nonlinear fractional multi-point boundary value problem at resonance. Int. J. Differ. Equ. 2011, Article ID 401803 (2011)

8. Khan, RA, Shah, K: Existence and uniqueness of solutions to fractional order multi-point boundary value problems. Commun. Appl. Anal. 19, 515-526 (2015)

9. Li, CF, Luo, XN, Zhou, Y: Existence of positive solutions of the boundary value problem for nonlinear fractional differential equations. Comput. Math. Appl. 59, 1363-1375 (2010)

10. LV, L, Wang, J, Wei, W: Existence and uniqueness results for fractional differential equations with boundary value conditions. Opusc. Math. 31, 629-643 (2011)

11. Miller, KS, Ross, B: An Introduction to the Fractional Calculus and Fractional Differential Equations. Wiley, New York (1993)

12. Rehman, M, Khan, RA: Existence and uniqueness of solutions for multi-point boundary value problems for fractiona differential equations. Appl. Math. Lett. 23(9), 1038-1044 (2010)

13. Yang, W: Positive solution to nonzero boundary values problem for a coupled system of nonlinear fractional differential equations. Comput. Math. Appl. 63, 288-297 (2012)

14. Caputo, M: Linear models of dissipation whose $Q$ is almost frequency independent. Geophys. J. R. Astron. Soc. 13(5) 529-539 (1967)

15. Hilfer, R: Applications of Fractional Calculus in Physics. World Scientific, Singapore (2000)

16. Kilbas, AA, Marichev, OI, Samko, SG: Fractional Integrals and Derivatives: Theory and Applications. Gordon \& Breach, Yverdon (1993)

17. Kilbas, AA, Srivastava, HM, Trujillo, JJ: Theory and Applications of Fractional Differential Equations. North-Holland Mathematics Studies, vol. 204. Elsevier, Amsterdam (2006)

18. Lakshmikantham, V, Leela, S, Vasundhara, J: Theory of Fractional Dynamic Systems. Cambridge Academic Publishers, Cambridge (2009)

19. Podlubny, I: Fractional Differential Equations, Mathematics in Science and Engineering. Academic Press, New York (1999)

20. Cai, L, Wu, J: Analysis of an HIV/AIDS treatment model with a nonlinear incidence rate. Chaos Solitons Fractals 41(1), 175-182 (2009)

21. Ahmad, B, Nieto, JJ: Existence results for a coupled system of nonlinear fractional differential equations with three-point boundary conditions. Comput. Math. Appl. 58, 1838-1843 (2009)

22. Su, X: Boundary value problem for a coupled system of nonlinear fractional differential equations. Appl. Math. Lett. 22, 64-69 (2009)

23. Shah, K, Khan, RA: Existence and uniqueness of positive solutions to a coupled system of nonlinear fractional order differential equations with anti-periodic boundary conditions. Differ. Equ. Appl. 7(2), 245-262 (2015)

24. Zeidler, E: Nonlinear Functional Analysis an Its Applications. I: Fixed Point Theorems. Springer, New York (1986)

25. Ahmad, B, Nieto, JJ: Existence of solutions for anti-periodic boundary value problems involving fractional differential equations via Leray-Schauder degree theory. Topol. Methods Nonlinear Anal. 35, 295-304 (2010)

26. Chen, T, Liu, W, Hu, Z: A boundary value problem for fractional differential equation with $p$-Laplacian operator at resonance. Nonlinear Anal. 75, 3210-3217 (2012)

27. Wang, X, Wang, L, Zeng, Q: Fractional differential equations with integral boundary conditions. J. Nonlinear Sci. Appl. 8, 309-314 (2015)

28. Wang, J, Zhou, Y, Wei, W: Study in fractional differential equations by means of topological degree methods. Numer Funct. Anal. Optim. 33(2), 216-238 (2012)

29. Yang, $A, G$, W: Positive solutions of multi-point boundary value problems of nonlinear fractional differential equation at resonance. J. Korean Soc. Math. Educ., Ser. B Pure Appl. Math. 16, 181-193 (2009)

30. Shah, K, Khalil, H, Khan, RA: Investigation of positive solution to a coupled system of impulsive boundary value problems for nonlinear fractional order differential equations. Chaos Solitons Fractals 77, 240-246 (2015)

31. Yao, ZJ: New results of positive solutions for second-order nonlinear three-point integral boundary value problems. J. Nonlinear Sci. Appl. 8, 93-98 (2015)

32. Nanware, A, Dhaigude, DB: Existence and uniqueness of solutions of differential equations of fractional order with integral boundary conditions. J. Nonlinear Sci. Appl. 7, 246-254 (2014)

33. Granas, A, Dugundji, J: Fixed Point Theory. Springer, New York (2003)

34. Isaia, F: On a nonlinear integral equation without compactness. Acta Math. Univ. Comen. 75, 233-240 (2006)

35. Deimling, K: Nonlinear Functional Analysis. Springer, New York (1985) 